Proceedings

\title{
Investigation of Particle Steering for Different Cylindrical Permanent Magnets in Magnetic Drug Targeting ${ }^{\dagger}$
}

\author{
Angelika S. Thalmayer *, Samuel Zeising, Georg Fischer and Jens Kirchner \\ Institute for Electronics Engineering, Friedrich-Alexander-Universität Erlangen-Nürnberg (FAU), \\ 91058 Erlangen, Germany; samuel.zeising@fau.de (S.Z.); georg.fischer@fau.de (G.F.); jens.kirchner@fau.de (J.K.) \\ * Correspondence: angelika.thalmayer@fau.de \\ + Presented at the 7th Electronic Conference on Sensors and Applications, 15-30 November 2020; Available \\ online: https:/ / ecsa-7.sciforum.net/.
}

Published: 15 November 2020

\begin{abstract}
Magnetic Drug Targeting is a promising cancer treatment that offers the possibility of increasing therapeutic efficiency while reducing the patient's side-effects. Thereby, the cancer-drug is bounded to magnetic nanoparticles, which are injected into a vessel and guided through the cardiovascular system into the tumor by an external magnetic field. However, a successful navigation depends on several multiphysical parameters including the properties of the nanoparticles, the flow characteristics of blood, and the gradient of the applied magnetic field. To investigate their impact, the propagation of particle packets within a $45^{\circ}$ bifurcation vessel was modeled in COMSOL Multiphysics ${ }^{\circledR}$. Therefore, magnets with varying radius to length ratios and magnetization (radial and axial) were placed right before the bifurcation. Furthermore, different fluid velocities additional to the influence of the gravitational force were evaluated. Overall, a strong dependency of the particle steering on the fluid velocity and the magnet's radius to length ratio was observed. Moreover, a radial magnetization has a greater impact on the particle propagation, while the gravitation can be neglected for higher velocities. However, when a single permanent magnet is used, the results depict that it is a fine line between deflecting or trapping a particle at the vessel wall.
\end{abstract}

Keywords: magnetic drug targeting; particle steering; permanent magnet

\section{Introduction}

Magnetic nanoparticles offer numerous applications in medical diagnosis and therapy, like magnetic drug targeting (MDT), which is a promising modern approach in cancer therapy [1]. Thereby, a cancer-drug is bound to magnetic nanoparticles. These particles are injected arterially near the tumor. As the nanoparticles have additionally magnetic properties, they can be guided to the tumorous tissue and trapped there by using an external magnetic field [2]. This enables a local chemotherapeutic treatment, which is advantageous in comparison to the traditional chemotherapy as the desired drug dose in the tumor can be adjusted individually. Thus, the chemotherapeutic drug dosage in the tumorous tissue can be increased, while at the same time the overall dosage in the patient is decreased, resulting in reduced side-effects [3].

However, the effectiveness of the treatment strongly depends on the accumulation of the nanoparticles in the tumor and, therefore, on the successful navigation of the particles through the cardiovascular system. The particle steering is influenced by several multiphysical parameters including the properties of the nanoparticles, the flow characteristics of the blood, and especially the gradient of the applied magnetic field [4]. In the literature, usually a Y-shaped vessel is investigated 
for the particle steering, since this Y-shape shows a symmetrical velocity distribution [4]. However, in the proposed paper, a COMSOL model, evaluating the propagation of particles packets within a vessel of $45^{\circ}$ bifurcation, was set up for different magnetic fields. In order to obtain a better overview of the multiphysical factors, the particle propagation for different blood velocities, all in a realistic range according to [5], were investigated. To analyze the influence of the magnetic field, the ratios of radius to length as well as magnetization direction (radial and axial) of a cylindrical NdFeB magnet were varied. Furthermore, the impact of the gravitational force was investigated.

\section{Fundamentals}

\subsection{Nanoparticles}

In MDT usually superparamagnetic iron oxide nanoparticles (SPIONs) are used as drug delivery carriers [6]. These particles consist of a small iron oxide core and, thus, are only composed of a single magnetic domain. Therefore, the SPIONs show no hysteretic behavior [7]. This missing remanent magnetization is advantageous for clinical applications, as the particles do not accumulate and, therefore, the risk of obstructing blood vessels is reduced. However, the SPIONs are not biocompatible [8] or stable in blood in general [9]. Thus, the iron oxide core is coated e. g. by lauric acid layers [9].

\subsection{Forces}

There are several forces affecting the SPIONs in MDT, like the magnetic, hydrodynamic drag, gravitational, buoyant, lift, and adhesion force $[4,10]$. However, for low flow regimes (low Reynolds numbers) and due to the fact that the applied nanoparticles are superparamagnetic and small in size, the most relevant forces are the (hydrodynamic) drag and the magnetic force [10,11]. The motion of one SPION in a vessel can be described using Newton's second law [11]

$$
m_{\mathrm{p}} \frac{\mathrm{d} v_{\mathrm{p}}}{\mathrm{d} t}=F_{\mathrm{m}}+F_{\mathrm{f}}
$$

Next to the particle mass $m_{\mathrm{p}}$ and the particle velocity $v_{\mathrm{p}}, F_{\mathrm{m}}$ corresponds to the magnetic and $F_{\mathrm{f}}$ to the drag force. The magnetic force on one single spherical particle mainly depends on the particle's volume and on the gradient of the magnetic field $H$ evaluated at the particle's position [11]

$$
F_{\mathrm{m}}=\frac{4 \pi r_{\mathrm{p}}^{3}}{3} \frac{\mu_{0} 3\left(\chi_{\mathrm{p}}-\chi_{\mathrm{f}}\right)}{3+\left(\chi_{\mathrm{p}}-\chi_{\mathrm{f}}\right)} H \cdot \nabla H
$$

where $r_{\mathrm{p}}$ is the particle radius and $\mu_{0} \approx 4 \pi \cdot 10^{-7} \mathrm{~N} / \mathrm{A}^{2}$ is the permeability of vacuum. Moreover, $\chi_{\mathrm{p}}$ and $\chi_{\mathrm{f}}$ corresponds to the susceptibility of the particle and the fluid, respectively. The permeabilities of the particle and fluid can be derived from

$$
\mu_{\mathrm{p}, \mathrm{f}}=\left(1+\chi_{\mathrm{p}, \mathrm{f}}\right) \mu_{0} .
$$

The drag force of one spherical particle is based on Stokes' law and can be described by

$$
F_{\mathrm{f}}=-6 \pi \eta r_{\mathrm{p}}\left(v_{\mathrm{p}}-v_{\mathrm{f}}\right),
$$

where $\eta$ is the fluid viscosity, and $v_{\mathrm{p}}$ and $v_{\mathrm{f}}$ are the particle and the fluid velocity, respectively [4].

\section{Materials and Methods}

To investigate the steering of the nanoparticles, a 3D model of a vessel with a $45^{\circ}$ bifurcation was set up in COMSOL Multiphysics ${ }^{\circledR}$. Right before the intersection, a cylindrical permanent magnet of 
rare earth $(\mathrm{NdFeB})$ was placed at a fixed position, depicted in Figure 1. The SPIONs were distributed in packets of $5 \times 100$ particles according to the density of the parabolic velocity profile. The fixed simulation parameters are summarized in Table 1. Moreover, the mesh sizes of the domains were optimized. For reasons of simplification, a newtonian fluid (water) was investigated for the fluid.

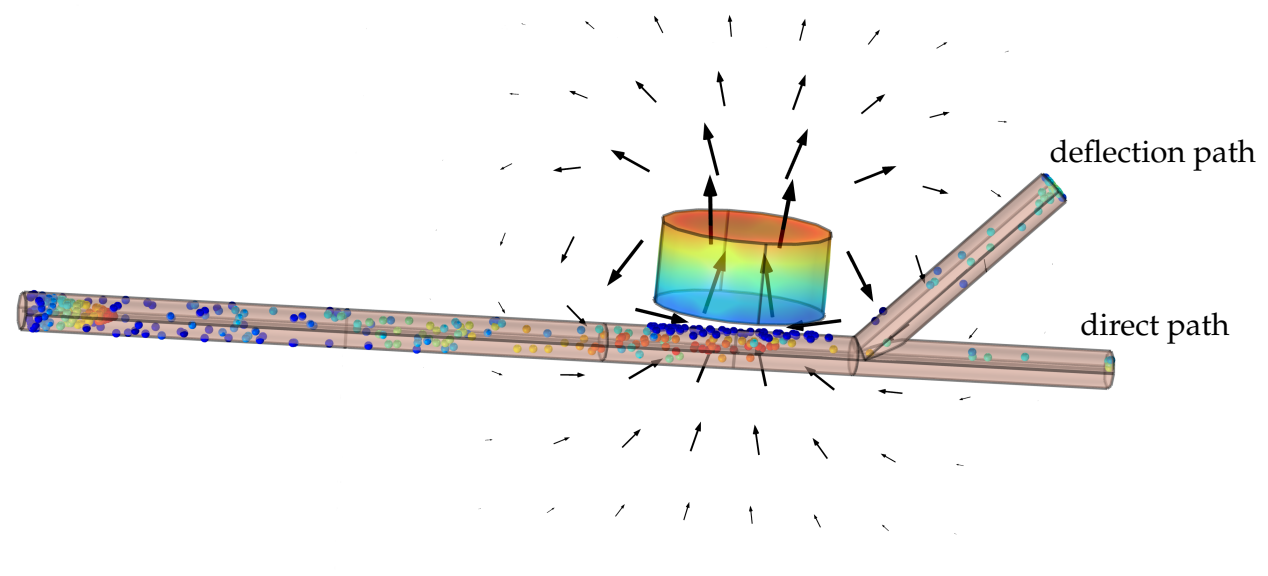

Figure 1. Transport of the SPIONs from the left to the right side within a $45^{\circ}$ bifurcation vessel. The cylindrical permanent magnet is placed outside the vessel applying the magnetic field. The velocity of one particle is depicted by its color; red corresponds to a high normalized velocity, whereas blue represents a low normalized particle velocity.

Table 1. Fixed simulation parameters.

\begin{tabular}{lllll}
\hline Category & Symbol & Value & Unit & Label \\
\hline \multirow{4}{*}{ vessel } & $r_{\mathrm{v}}$ & 2 & $\mathrm{~cm}$ & radius \\
& $L$ & 13 & $\mathrm{~cm}$ & $\begin{array}{l}\text { length } \\
\text { relative permeability of the fluid }\end{array}$ \\
& $\mu_{\mathrm{f}}$ & 1 & 1 & relation \\
particle & $r_{\mathrm{p}}$ & 350 & $\mathrm{~nm}$ & radius \\
& $\rho$ & 2000 & $\mathrm{~kg} / \mathrm{m}^{3}$ & density \\
& $\mu_{\mathrm{p}}$ & 4000 & 1 & relative permeability \\
\hline \multirow{2}{*}{ magnet } & $V$ & 3 & $\mathrm{~cm}^{3}$ & volume \\
& $M_{\mathrm{sat}}$ & $10^{6}$ & $\mathrm{~A} / \mathrm{m}$ & saturation magnetization \\
\hline
\end{tabular}

Overall, two different scenarios were investigated. In the first one, the velocity of the background flow was varied to be $3 \mathrm{~mL} / \mathrm{min}, 6 \mathrm{~mL} / \mathrm{min}, 12 \mathrm{~mL} / \mathrm{min}$, and $24 \mathrm{~mL} / \mathrm{min}$, respectively, all chosen to be realistic for the human blood according to [5]. Furthermore, the influence of the gravitational force was taken into account. In the second scenario, the influence of the magnetic field was analyzed, considering varying radius to length ratios ( $\mathrm{rtl})$ of the magnet $(0.5,1$ and 2$)$, while the volume as well as the distance between the magnet, the intersection and the vessel wall, stayed the same. Next to a radial magnetization, an axial magnetization of the magnet was investigated. For the axial magnetization, the magnet was rotated by $90^{\circ}$ so that the direction of the magnetic field lines stayed unchanged. In the second scenario, the gravitational force was not considered. For both scenarios, the particle distribution in the desired deflection and the direct path was studied. The simulation time was chosen such that every particle reaches the end of the tube. 


\section{Results and Discussion}

\subsection{Influence of the Fluid Velocity}

Before analyzing the particle steering due to the external magnetic field, the fundamental impact of the fluid velocity should be clarified. Therefore, the normalized fluid velocity profiles of $v=3 \mathrm{~mL} / \mathrm{min}$ and $v=24 \mathrm{~mL} / \mathrm{min}$ are displayed in Figure 2. Until the bifurcation, the velocity profiles are both parabolic and the normalized amplitude decreases after the intersection according to the continuity equation. For an applied velocity of $v=3 \mathrm{~mL} / \mathrm{min}$, the velocity after the bifurcation in both, the direct and the deflection paths, is equal. For $v=6 \mathrm{~mL} / \mathrm{min}$ the ratio of deflection to direct path velocity is $1: 1.09$, for $v=12 \mathrm{~mL} / \mathrm{min} 1: 1.27$ and for $v=24 \mathrm{~mL} / \mathrm{min} 1: 1.78$, respectively. However, at the bifurcation and shortly after it, turbulences occur, being intensified for faster velocities. These turbulences, in turn, affect the particles' propagation in the blood stream, since they influence the drag force. Figure 3 depicts, that for a fluid velocity of $3 \mathrm{~mL} / \mathrm{min}$ the particles distribute equally, whereas for an increasing fluid velocity more particles take the direct path and, thus, get not deflected.
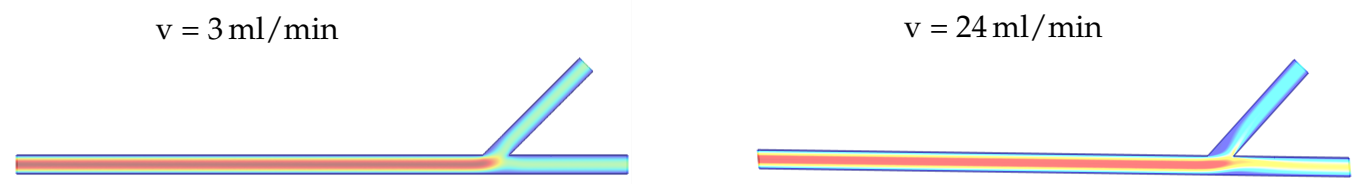

Figure 2. Normalized velocity profile of the setup. The red color corresponds to a high normalized velocity, whereas blue represents a low normalized velocity.
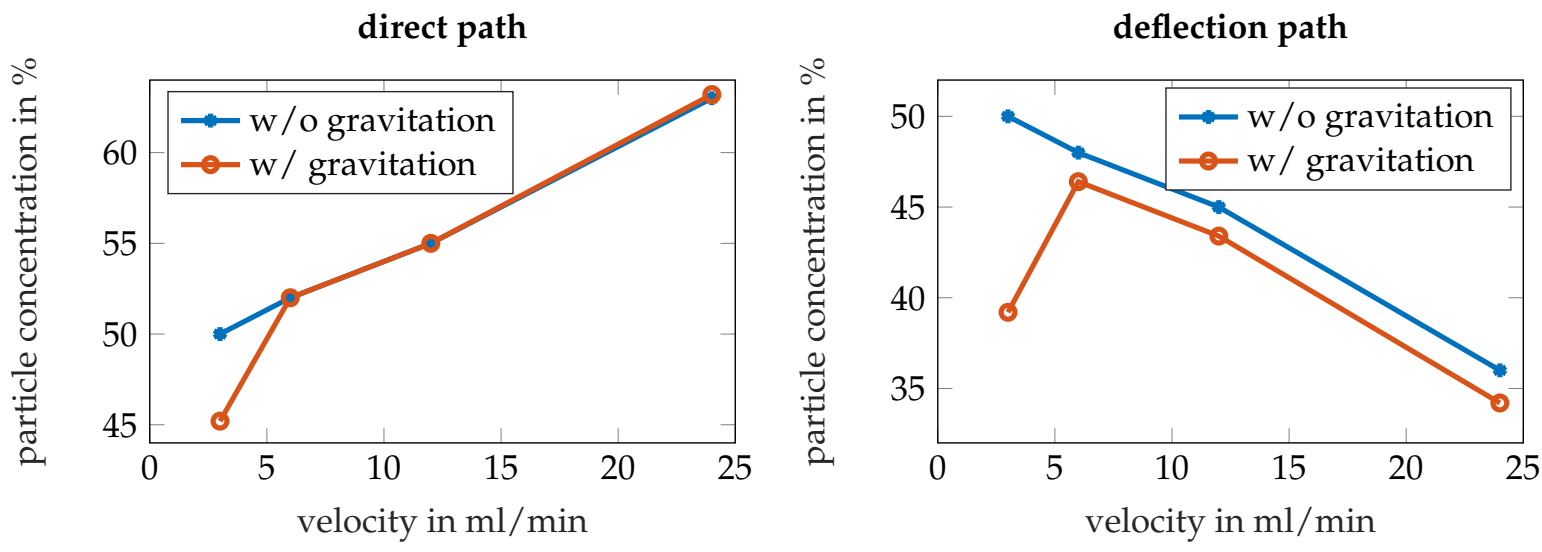

Figure 3. Comparison of the particle concentration in the two paths without an external applied magnetic field and with (w/) and without (w/o) the gravitational force.

Moreover, due to the parabolic velocity profile, the drag force in the middle of the vessel is stronger than at the boundary and in consequence, the particles in the middle move faster than the ones closer to the boundary. This issue can be observed in Figure 1. At the left entrance of the vessel, a new particle packet is injected. The parabolic velocity profile can be observed very clearly in the particle distribution. Furthermore, the figure shows that the new injected particles already overtakes the slower particles of the previous packet. This effect was also observed in the measurement results of [12], where the distribution of the particles, used for communication, resulted in intersymbol interference. 


\subsection{Influence of the Gravitational Force}

To investigate the influence of the gravitational force, the propagation of the particle packages was studied with $(\mathrm{w} /)$ and without $(\mathrm{w} / \mathrm{o})$ considering the gravitational force for all four applied blood velocities, according to the first scenario, introduced in Section 3. The particle concentration of the direct and the deflection path is illustrated in Figure 3. As aforementioned, without the gravitational force, the particles distribute equally for low particle velocities. However, with an increased input velocity less particles take the upper deflection path.

Figure 3 depicts that the influence of the gravitational force decreases with an increasing fluid velocity. An impact in the direct path is only observable for $v=3 \mathrm{~mL} / \mathrm{min}$. Simultaneously, the influence in the deflection path is the highest for $v=3 \mathrm{~mL} / \mathrm{min}$. However, for higher velocities the curves with and without gravitation are approximately parallel. According to Equation (4), the drag force is proportional to the velocity and, therefore, its impact on the particles increases. However, if gravitation is considered, for every fluid velocity some particles remain in the vessel. These ones are especially the particles that are injected at the lower boundary of the vessel, since, due to the parabolic velocity profile, the fluid velocity at the boundaries is $v=0$. Therefore, the drag force on this particles is lower than the gravitational force and, thus, they are not able to propagate through the vessel.

\subsection{Influence of the Magnet}

The particle concentrations for the second scenario are shown in Figure 4. The results depict that for lower velocities the applied magnetic field is too strong. In consequence, most of the particles are trapped by the magnet and, therefore, are stuck in the vessel. This particle trapping is also visible in Figure 1. However, as aforementioned, the drag force increases with the velocity, resulting in a decreased impact of the magnetic field on the SPIONs deflection.
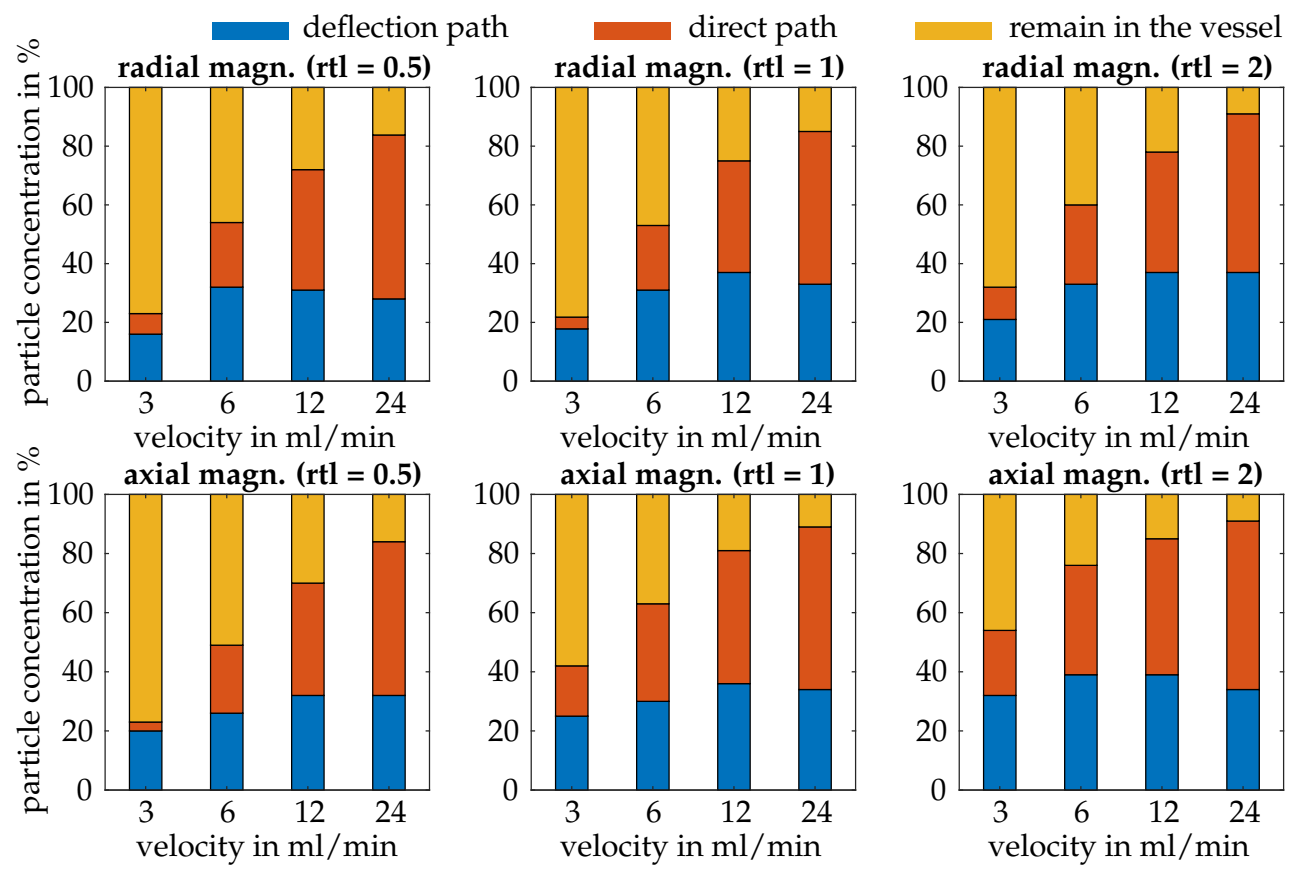

Figure 4. Results of the steering performance over the fluid velocity for the different applied permanent magnets (varied radius to length ratios (rtl)). The first row displays the results for the radial magnetization, whereas the second corresponds to the axial ones.

By comparing the different magnetization directions, the results depict that the impact of the magnet is higher for a radial magnetization. Additionally, a smaller rtl-value has a greater influence on the particle propagation. The reason for this issue can be found in Equation (2): The magnetic force 
on the particle depends on the gradient of the magnetic field. As for higher rtl-values, the gradient of the magnetic field is smaller, its impact on the particles decreases.

Finally, the way of one single nanoparticle through the vessel was investigated in detail. The particles, which are injected in the upper part of the vessel, are trapped by the magnet, while the ones in the lower middle are guided to the desired deflection path. The lower injected particles stay in the direct path. Therefore, it can be concluded that for every setup of a fix fluid velocity and magnet, there must be an optimal zone to guide the SPIONs in a predefined direction. Thus, the particle deflecting could be prepared even before the bifurcation. If the SPIONs are located in the optimal zone, they will be properly deflected automatically.

\section{Conclusions}

The paper proposes that the particle steering strongly depends on the fluid velocity and the applied magnetic field. Due to the high dynamic range of the human blood velocity [5], the particle propagation strongly differs even without an additional magnetic field. For the chosen nanoparticles, the gravitational force only has a notable influence for $v=3 \mathrm{~mL} / \mathrm{min}$. In general, a deflection of the particles towards a desired direction is difficult by using only one simple permanent magnet. Especially for low fluid velocities, most of the particles get trapped. On the contrary, for $v=24 \mathrm{~mL} / \mathrm{min}$ the magnet should be stronger. Since the influence of the blood velocity is that strong, the current velocity should be known and the magnetic field strength should be chosen accordingly. Therefore, in future studies the permanent magnet will be replaced by an electromagnet, which will allow to fit the applied magnetic field strength and its gradient to the current fluid velocity. To solve the trapping problem, the magnet can be switched on and off, as suggested by [4]. Moreover, an additional magnet on the other side of the vessel is an option, pulling the trapped particles away from the vessel wall towards the region of a higher velocity. However, in the real human body, this is not always possible.

Funding: This research received no external funding.

Conflicts of Interest: The authors declare no conflict of interest.

\section{References}

1. Pankhurst, Q.A.; Connolly, J.; Jones, S.K.; Dobson, J. Applications of magnetic nanoparticles in biomedicine. J. Phys. D Appl. Phys. 2003, 36, R167.

2. Tietze, R.; Lyer, S.; Dürr, S.; Struffert, T.; Engelhorn, T.; Schwarz, M.; Eckert, E.; Göen, T.; Vasylyev, S.; Peukert, W.; et al. Efficient drug-delivery using magnetic nanoparticles-biodistribution and therapeutic effects in tumour bearing rabbits. Nanomed. Nanotechnol. Biol. Med. 2013, 9, 961-971.

3. Alexiou, C.; Schmidt, A.; Klein, R.; Hulin, P.; Bergemann, C.; Arnold, W. Magnetic drug targeting: Biodistribution and dependency on magnetic field strength. J. Magn. Magn. Mater. 2002, 252, 363-366. doi:10.1016/S0304-8853(02)00605-4.

4. Hoshiar, A.K.; Le T.A.; Amin, F.U.; Kim, M.O.; Yoon, J. Studies of aggregated nanoparticles steering during magnetic-guided drug delivery in the blood vessels. J. Magn. Magn. Mater. 2017, 427, 181-187. doi:10.1016/j.jmmm.2016.11.016.

5. Klarhöfer, M.; Csapo, M.B.; Balassy, C.; Szeles, J.; Moser, E. High-resolution blood flow velocity measurements in the human finger. Magn. Reson. Med. 2001, 45. doi:10.1002/mrm.1096.

6. Hennig, T.L.; Unterweger, H.; Lyer, S.; Alexiou, C.; Cicha, I. Magnetic Accumulation of SPIONs under Arterial Flow Conditions: Effect of Serum and Red Blood Cells. Molecules 2019, 24, 2588. doi:10.3390/molecules24142588.

7. Cullity, B.D.; Graham, C.D. Introduction to Magnetic Materials; John Wiley \& Sons, Inc.: Hoboken, NJ, USA, 2008. doi:10.1002/9780470386323.

8. Hanini, A.; Schmitt, A.; Kacem, K.; Chau, F.; Ammar, S.; Gavard, J. Evaluation of iron oxide nanoparticle biocompatibility. Int. J. Nanomed. 2011, 6, 787-794. doi:10.2147/IJN.S17574. 
9. Zaloga, J.; Janko, C.; Nowak, J.; Matuszak, J.; Knaup, S.; Eberbeck, D.; Tietze, R.; Unterweger, H.; Friedrich, R.P.; Duerr, S.; et al. Development of a lauric acid/albumin hybrid iron oxide nanoparticle system with improved biocompatibility. Int. J. Nanomed. 2014, 9, 4847-4866. doi:10.2147/IJN.S68539.

10. Alnaimat, F.; Dagher, S.; Mathew, B.; Hilal-Alnqbi, A.; Khashan, S. Microfluidics Based Magnetophoresis: A Review. Chem. Rec. 2018, 18, 1596-1612. doi:10.1002/tcr.201800018.

11. Furlani, E.P.; Sahoo, Y. Analytical model for the magnetic field and force in a magnetophoretic microsystem. J. Phys. D Appl. Phys. 2006, 39, 1724-1732. doi:10.1088/0022-3727/39/9/003.

12. Wang, J.; Hu, D.; Shetty, C.; Hassanieh, H. Understanding and embracing the complexities of the molecular communication channel in liquids. In Proceedings of the 26th Annual International Conference on Mobile Computing and Networking, London, UK, 21-25 September 2020; ACM: New York, NY, USA, 2020; pp. 1-15. doi:10.1145/3372224.3419191.

Publisher's Note: MDPI stays neutral with regard to jurisdictional claims in published maps and institutional affiliations.

(C) 2020 by the authors. Licensee MDPI, Basel, Switzerland. This article is an open access article distributed under the terms and conditions of the Creative Commons Attribution (CC BY) license (http://creativecommons.org/licenses/by/4.0/). 